\title{
Behavior of magnetic currents in anisotropic Heisenberg spin chains out of equilibrium
}

\author{
V. Popkov, ${ }^{1,2}$ Mario Salerno, ${ }^{1}$ and G. M. Schütz ${ }^{3}$ \\ ${ }^{1}$ Dipartimento di Fisica “E.R. Caianiello," Università di Salerno, via ponte don Melillo, 84084 Fisciano (SA), Italy \\ ${ }^{2}$ Dipartimento di Fisica, Università di Firenze, via Sansone 1, 50019 Sesto Fiorentino (FI), Italy and \\ Max Planck Institute for the Physics of Complex Systems, 01187 Dresden, Germany \\ ${ }^{3}$ Institute of Complex Systems II, Forschungszentrum Jülich, Jülich, Germany
}

(Received 10 January 2012; revised manuscript received 4 March 2012; published 22 March 2012)

\begin{abstract}
The behavior of the magnetic currents in one-dimensional Heisenberg $X X Z$ spin chains kept out of equilibrium by boundary driving fields is investigated. In particular, the dependence of the spin currents on the anisotropy parameter $\Delta$ and on the boundary fields is studied both analytically and numerically in the framework of the Lindblad master equation formalism. We show that the spin current can be maximized with appropriate choices of the boundary fields, and for odd system sizes, $N$, we demonstrate the existence of additional symmetries that cause the current to be an odd function of $\Delta$. From direct numerical integrations of the quantum master equation, we find that for an arbitrary $N$ the current $J_{z}(N)$ vanishes for $\Delta=0$, while for $\Delta$ negative it alternates its sign with the system size. In the gapless critical region $|\Delta|<1$, the scaling of the current is shown to be $J_{z}(N) \sim 1 / N$ while in the gapped region $|\Delta|>1$ we find that $J_{z}(N) \sim \exp (-\alpha N)$. A simple mean-field approach, which predicts rather well the values of $J_{z}(N)$ for the gapped region and the values of the absolute current maxima in the critical region, is developed. The existence of two different stationary solutions for the mean-field density matrix in the whole parameter range is also demonstrated.
\end{abstract}

DOI: 10.1103/PhysRevE.85.031137

PACS number(s): 05.60.-k, 03.65.Yz, 75.10.Pq

\section{INTRODUCTION}

Recent advances in the experimental manipulation of nanowires and quantum dots have made it possible to investigate quantum systems consisting of a few quantum dots or quantum bits. On the other hand, manipulations of single quantum bits (e.g., application of a quantum gate) form the basis of the functioning of any elementary block of a quantum computing device. However, a theoretical understanding of microscopic quantum systems out of equilibrium (e.g., under constant pumping or continuous measurement by a quantum probe) is far from being complete, apart from the most simple cases such as a single two-level system or a quantum harmonic oscillator under external pumping or in contact with a reservoir. In view of this, the role of simple but nevertheless spatially extended systems, amenable to both analytic and numerical investigation, becomes important. In particular, it is desirable to understand the interplay between many-body bulk properties in extended quantum systems (e.g., magnon excitations or magnetization currents in quantum spin systems) and local (e.g., applied to a small part of a system) pumping driving the system constantly out of equilibrium.

To induce a nonequilibrium situation, one can couple the system at both ends to magnetization reservoirs at different potentials so to create magnetic gradients in the system. This is frequently done in the framework of the Lindblad master equation $[1,2]$ with reservoirs modeled by means of Lindblad operators acting at the system ends. A model of this type was recently considered in Ref. [3], where an $X X Z$ quantum chain of finite size and constant effective pumping at the first and the last site, which induces an effective gradient along the $z$ axis, was proposed and studied. In particular, a negative feedback of the boundary pumping on a conserved magnetization current beyond a certain amplitude of the gradient was observed.

We remark that transport of magnetization in the Heisenberg model has been extensively investigated during the past years (for extended reviews, see Refs. [4,5]) using a range of alternate methods, including Bethe-ansatz calculations [6,7], Lagrange multipliers [8,9], exact diagonalizations [10,11], the Lanczos method [12], quantum Monte Carlo [13], etc.

In the present study, we address the problem of how the current in a quantum chain of the type considered in Ref. [3], which we view as a generic string of quantum bits manipulated at the ends through a stationary pumping, can be maximized by a suitable choice of Lindblad operators. The model is conceptually simple. It describes a system of interacting two-level systems (quantum bits), and in the absence of pumping it possesses remarkable properties (integrability). In a nonequilibrium situation, the most natural quantities to consider are the stationary currents, these being the simplest many-body correlation functions and the simplest indicators of how far the system is from equilibrium. Our first objective is the study of the behavior of stationary conserved magnetization currents $J_{z}(N)$ in a system of size $N$, and to maximize their values with respect to the boundary pumping. In particular, we will demonstrate the following:

(i) The current $J_{z}(N)$ can be made at least one order of magnitude larger than in Ref. [3] with an appropriate choice of boundary conditions.

(ii) The current scales as $J_{z}(N) \sim 1 / N$ in the critical region $-1<\Delta<1$ and as $J_{z}(N) \sim \exp (-\alpha N)$ in the gapped region.

(iii) For arbitrary $N$, the current $J_{z}(N)$ always vanishes for $\Delta=0$ while for $\Delta$ negative it alternates its sign with the system size: $\operatorname{sgn}\left(J_{z}(N)\right)=(-1)^{N}$.

By neglecting nearest-neighbor correlations, we also develop a simple semiclassical (mean-field) approach that predicts in an excellent manner the current $J_{z}(N)$ in the gapped region $|\Delta|>1$ and gives a qualitatively good agreement for the absolute current maxima in the critical region $|\Delta|<1$. The existence of two different stationary solutions for the mean-field density-matrix equation is demonstrated for the 
whole parameter range, and the possibility that multiple stationary solutions can exist also in the quantum case is suggested. This last possibility represents a very interesting problem that deserves further investigations.

The plan of the paper is the following. In Sec. II, we introduce the model equation for our open spin chain and discuss the main properties of the master equation in the Lindblad form. In particular, we discuss the action of Lindblad operators at the chain boundaries and show how these operators can be used to induce gradients and boundary magnetization values along spatial directions. In Sec. III, we discuss and analytically prove the existence of a nontrivial symmetry of the Lindblad master equation with respect to the anisotropy parameter $\Delta \rightarrow-\Delta$ interchange for the case of chains with an odd number of spins. In Sec. IV, we present and discuss numerical quantum results obtained from direct integrations of the Lindblad master equation. Section V is devoted to the semiclassical approach and its comparisons with the quantum results. Finally, a brief summary of the main results of the paper and future perspectives are given in the Conclusion.

\section{MODEL EQUATIONS}

We study the quantum master equation in the Lindblad form for the $X X Z$ model with a drive at the boundaries of the form

$$
\begin{aligned}
\frac{\partial \rho}{\partial t}= & -i[H, \rho]-\frac{1}{2} \sum_{m=1}^{4}\left\{\rho, L_{m}^{\dagger} L_{m}+V_{m}^{\dagger} V_{m}+W_{m}^{\dagger} W_{m}\right\} \\
& +\sum_{m=1}^{4}\left(L_{m} \rho L_{m}^{\dagger}+V_{m} \rho V_{m}^{\dagger}+W_{m} \rho W_{m}^{\dagger}\right)
\end{aligned}
$$

(here and below we set $\hbar=1$ and denote with a dagger the adjoint operator). In this approach [1], $\rho$ is the reduced density matrix and $H$ is the Hamiltonian of the open $X X Z$ chain,

$$
H=J_{E} \sum_{k=1}^{N-1} \sigma_{k}^{x} \sigma_{k+1}^{x}+\sigma_{k}^{y} \sigma_{k+1}^{y}+\Delta \sigma_{k}^{z} \sigma_{k+1}^{z} .
$$

The dissipative terms of the Lindblad master equation contain three sets of the Lindblad operators $L_{m}, V_{m}$, and $W_{m}$, which we choose to act locally at the open ends (the first and the last site) of the quantum chain. The Lindblad operators acting on the first site $k=1$ carry subscripts $m=1,2$ and are given explicitly by

$$
\begin{aligned}
L_{1}=\alpha\left(\sigma_{1}^{x}+i \sigma_{1}^{y}\right), & L_{2}=\beta\left(\sigma_{1}^{x}-i \sigma_{1}^{y}\right), \\
V_{1}=p\left(\sigma_{1}^{y}+i \sigma_{1}^{z}\right), & V_{2}=q\left(\sigma_{1}^{y}-i \sigma_{1}^{z}\right), \\
W_{1}=u\left(\sigma_{1}^{z}+i \sigma_{1}^{x}\right), & W_{2}=v\left(\sigma_{1}^{z}-i \sigma_{1}^{x}\right) .
\end{aligned}
$$

The Lindblad operators $L_{m}, V_{m}$, and $W_{m}$ with $m=3,4$ act on the last site $i=N$ and are given by the expressions for $L_{m-2}, V_{m-2}$, and $W_{m-2}$, respectively, with $\alpha, \beta, p, q, u, v$ substituted by $\alpha^{\prime}, \beta^{\prime}, p^{\prime}, q^{\prime}, u^{\prime}, v^{\prime}$. We can always choose the constants $\alpha, \beta, \ldots, v^{\prime}$ to be real and non-negative, since in Eq. (1) all Lindblad operators appear in pairs. The operators $L_{m}, V_{m}$, and $W_{m}$, taken alone, target polarization along the axes $z, x$, and $y$, respectively, on the boundary sites. Notice that even in the free-fermion case $\Delta=0$, this generalized Lindblad dynamics is not integrable, as is the case in Ref. [14], because the Lindblad operators are not all quadratic in fermionic variables.

It is easy to verify from (1) that $\frac{\partial}{\partial t} \operatorname{Tr}(\rho)=0, \rho^{\dagger}=\rho$, $\frac{\partial}{\partial t} \operatorname{Tr}\left(\rho^{2}\right) \neq 0$. The first two relations are necessary for interpreting $\rho$ as a density matrix with $\operatorname{Tr}(\rho)=1$, while the latter means that we have an open system: an initially pure state $\rho=|\phi\rangle\langle\phi|$ will not remain pure over the course of time. In contrast to reduced density matrices of isotropic ferromagnetic Heisenberg chains or many-body systems on complete graphs, which can be fully characterized (see Refs. [15,16]), stationary density matrices of an open quantum system out of equilibrium [in this context, solutions of Eq. (1)] are largely unknown (see Ref. [1] for a review). The action of the Lindblad operators acting on the first site $\left(L_{m}, V_{m}\right.$, and $W_{m}$ with $\left.m=1,2\right)$ becomes clear if we write down the equations of motion for the expectation values of boundary operators $\sigma_{1}^{x}, \sigma_{1}^{y}, \sigma_{1}^{z}$. Using the master equation and the properties of the Pauli matrices $\sigma^{k} \sigma^{j} \sigma^{k}=-\sigma^{j}$ for $j \neq k$ and $\sigma^{i} \sigma^{j}=i \varepsilon_{i j k} \sigma^{k}$ for $i \neq j$, one obtains after some algebra

$$
\begin{aligned}
& \frac{d\left\langle\sigma_{1}^{z}\right\rangle}{d t}=H_{\mathrm{part}}^{z}-\Gamma_{z}\left(\left|\sigma_{1}^{z}\right\rangle-\sigma_{L}^{z}\right), \\
& \frac{d\left\langle\sigma_{1}^{x}\right\rangle}{d t}=H_{\mathrm{part}}^{x}-\Gamma_{x}\left(\left\langle\sigma_{1}^{x}\right\rangle-\sigma_{L}^{x}\right), \\
& \frac{d\left\langle\sigma_{1}^{y}\right\rangle}{d t}=H_{\mathrm{part}}^{y}-\Gamma_{y}\left(\left|\sigma_{1}^{y}\right\rangle-\sigma_{L}^{y}\right),
\end{aligned}
$$

where $H_{\text {part }}^{k}$ denotes the term $-i \operatorname{Tr}\left(\sigma_{1}^{k}[H, \rho]\right)$ with $k=x, y, z$ and

$$
\begin{aligned}
& \Gamma_{z}=K+\left(2 \alpha^{2}+2 \beta^{2}\right), \quad \sigma_{L}^{z}=\frac{4 \alpha^{2}-4 \beta^{2}}{\Gamma_{z}} \\
& \Gamma_{x}=K+\left(2 p^{2}+2 q^{2}\right), \quad \sigma_{L}^{x}=\frac{4 p^{2}-4 q^{2}}{\Gamma_{x}} \\
& \Gamma_{y}=K+\left(2 u^{2}+2 v^{2}\right), \quad \sigma_{L}^{y}=\frac{4 u^{2}-4 v^{2}}{\Gamma_{y}} \\
& K=2\left(p^{2}+q^{2}+u^{2}+v^{2}+\alpha^{2}+\beta^{2}\right) .
\end{aligned}
$$

If $\Gamma_{x}, \Gamma_{y}, \Gamma_{z}$ are sufficiently large with respect to the exchange constant $J_{E}$ of the Heisenberg Hamiltonian, the Hamiltonian part, $H_{\text {part }}$, in the above equations is negligible and we expect the averages $\left\langle\sigma_{1}^{i}(t)\right\rangle$ to relax to their "left boundary" values $\sigma_{L}^{i}$,

$$
\left\langle\sigma_{1}^{i}(t)\right\rangle=\sigma_{L}^{i}+\left\langle\sigma_{1}^{i}(0)-\sigma_{L}^{i}\right\rangle e^{-\Gamma_{i} t},
$$

after a relaxation time of order $1 / \Gamma_{i}$. Notice that $\left|\sigma_{L}^{i}\right| \leqslant 1$ for any choice of $p, q, u, v, \alpha, \beta$. For the right boundary, we will obtain the equations of motion for expectation values $\left\langle\sigma_{k=N}^{i}\right\rangle$ by substituting $\alpha, \beta \rightarrow \alpha^{\prime}, \beta^{\prime}$, etc., in (4), from which we see that the right boundary values $\sigma_{R}^{i}$ are given by the ratios of the Lindblad operator amplitudes for $m=3,4$. Thus, by choosing a specific set of Linblad operators $L_{m}, V_{m}$, and $W_{m}$, we may induce gradients and boundary magnetization values along the axes $x, y$, and $z$, respectively. We remark, however, that not all sets of possible boundary values $\sigma_{L}^{x}, \sigma_{L}^{y}$, and $\sigma_{L}^{z}$ are accessible. For instance, if $\Gamma_{x}>2 p^{2}+2 q^{2}$, meaning that 
some of the constants $u, v, \alpha, \beta$ are nonzero, the maximal value of $\sigma_{L}^{x}$ (attained by the choice $q=0$ ) is $\sigma_{L}^{x}=4 p^{2} / \Gamma_{x}<1$. Note that the condition $\left(\sigma_{L}^{x}\right)^{2}+\left(\sigma_{L}^{y}\right)^{2}+\left(\sigma_{L}^{z}\right)^{2} \leqslant 1$ guarantees the positiveness of the steady-state reduced density matrix $\rho_{1}=\operatorname{Tr}_{2,3, \ldots, N} \rho$ of the left boundary site $i=1$ in the case of infinitely large coupling constants $\Gamma_{\alpha}$, the equality sign corresponding to a pure one-site state $\operatorname{Tr}\left(\rho_{1}\right)^{2}=1$.

For our study, we choose the following set of the Lindblad operators: only three operators $V_{1}, V_{4}$, and $W_{4}=v^{\prime}\left(\sigma_{N}^{z}-i \sigma_{N}^{x}\right)$ are nonzero and have the following amplitudes:

$$
p=\sqrt{\Gamma}, \quad q^{\prime}=\sqrt{0.5 \Gamma}, \quad v^{\prime}=\sqrt{\Gamma},
$$

while all the remaining constants $\alpha, \beta, \ldots, u^{\prime}$ are set to zero. With the choice (7), we set "desirable" boundary magnetization values to $\sigma_{L}^{y}=0, \sigma_{L}^{z}=0, \sigma_{L}^{x}=1$ at the left boundary and $\sigma_{L}^{y}=-0.8, \sigma_{L}^{z}=0, \sigma_{L}^{x}=-0.5$ at the right boundary, as can be verified straightforwardly from (5). The coupling constant $\Gamma$ will be set to $\Gamma=4 J_{E}$ in most cases, so that the amplitudes of the Lindblad operators and the Heisenberg exchange interaction are comparable. The choice (7) can be argued to be a "minimal" favorable choice in order to produce the largest possible stationary current along the the anisotropy axis $z$,

$$
J_{z}=\sigma_{n}^{y} \sigma_{n+1}^{x}-\sigma_{n}^{x} \sigma_{n+1}^{y}
$$

(as a conserved quantity, it does not depend on $n$ ). It is worth remarking that for our choice of Lindblad operators, the averaged energies per site of nonequilibrium steady states are very small, this corresponding to a very high (practically infinite) temperature.

Notice that from a physical point of view, our choice of Lindblad operators corresponds to magnetic reservoirs with easy-plane magnetizations at the boundaries. This could be realized by means of twisted magnetic fields at the boundary with the twist angle fixed by the parameters in Eq. (7). This setting is "orthogonal" to the choice made in Ref. [3], where the boundary gradient with the help of Lindblad operators $L_{m}$ was set along the anisotropy axis. In our case, we have no gradient along the conserved direction, $\sigma_{L}^{z}=\sigma_{R}^{z}=0$, while strong gradients $1 \rightarrow-0.5$ and $0 \rightarrow-0.8$ are set along the perpendicular directions. Note that an increase of a gradient in one (say, $X$ ) component has the expense of decreasing a gradient in the other component; see (5). For example, it is not possible, by keeping nonzero $V_{1}, V_{4}$, and $W_{4}$ with arbitrary (but nonzero) amplitudes (3), to realize the maximal gradients $1 \rightarrow-1$ in both the $y$ and $x$ directions.

\section{SYMMETRY RELATIONS FOR ODD $N$}

For odd $N$, the master equation in the Lindblad form (1) has a nontrivial symmetry with respect to $\Delta \rightarrow-\Delta$ interchange, described below. By a unitary transformation, $U=\sigma_{2}^{z} \sigma_{4}^{z} \cdots \sigma_{N-1}^{z}=U^{\dagger}$, the Hamiltonian $H$ transforms as $U H(\Delta) U^{\dagger}=-H(-\Delta)$, while the Lindblad operators do not transform since they are acting on the boundary sites $i=1, N$.

Applying $U \cdots U^{\dagger}$ to the master equation (1), and taking the complex conjugate, we obtain Eq. (1) for the transformed density matrix $\rho^{\prime}=U \rho^{*}(-\Delta) U^{\dagger}$ and Lindblad operators $L_{i}^{*}, V_{i}^{*}, W_{i}^{*}$. In the basis where $\sigma^{x}$ is diagonal, we obtain
$L_{i}^{*}=L_{i}^{\dagger}, V_{i}^{*}=V_{i}$, and $W_{i}^{*}=-W_{i}$. For our choice of the Lindblad operators (7) not containing operators $L$, the resulting Lindblad equation for $\rho^{\prime}$ is identical to the original one, with a replacement $\Delta \rightarrow-\Delta$, yielding the symmetry

$$
\rho(\Delta, t)=U \rho^{*}(-\Delta, t) U
$$

This symmetry implies, in particular, the sign change for one-point functions along the anisotropy axis $\left\langle\sigma_{n}^{z}(-\Delta)\right\rangle=$ $-\left\langle\sigma_{n}^{z}(\Delta)\right\rangle$, and site-to-site alternations along the other axes $\left.\left\langle\sigma_{n}^{y}(-\Delta)\right\rangle=(-1)^{n+1}\left\langle\sigma_{n}^{y}(\Delta)\right\rangle, \sigma_{n}^{x}(-\Delta)\right\rangle=(-1)^{n+1}\left\langle\sigma_{n}^{x}(\Delta)\right\rangle$. Conserved current expectation $\left\langle J_{z}(n)\right\rangle=\left\langle\sigma_{n}^{y} \sigma_{n+1}^{x}-\sigma_{n}^{y} \sigma_{n+1}^{x}\right\rangle$ changes sign under $\Delta$ sign reversal,

$$
\left\langle J_{z}(n)\right\rangle(N, \Delta)=-\left\langle J_{z}(n)\right\rangle(N,-\Delta) \quad \text { for odd } N .
$$

The sign alternation produces interesting consequences for the static structure factors $S^{\alpha \beta}(k, \Delta)=$ $\sum_{n<m} e^{i k(m-n)}\left\langle\sigma_{n}^{\alpha} \sigma_{n+1}^{\beta}\right\rangle$. Such structure factors can be used as entanglement witnesses [17,18] and are experimentally accessible via neutron scattering. Using (9), we obtain

$$
\begin{aligned}
S^{z z}(k, \Delta) & =S^{z z}(k,-\Delta), \\
S^{\alpha \alpha}(0, \Delta) & =S^{\alpha \alpha}(\pi,-\Delta) \quad \text { for } \quad \alpha=y, x .
\end{aligned}
$$

\section{NUMERICAL RESULTS}

We integrate numerically in time the full set of equations for the reduced density-matrix elements (1), starting from the maximally mixed initial state. Once the convergence to the stationary state has been reached, we measure the current $\left\langle J_{z}(n)\right\rangle=\left\langle\sigma_{n}^{y} \sigma_{n+1}^{x}-\sigma_{n}^{y} \sigma_{n+1}^{x}\right\rangle=\operatorname{Tr}\left(\rho J_{z}(n)\right)$ and stationary magnetization profiles $\left\langle\sigma_{n}^{i}\right\rangle=\operatorname{Tr}\left(\rho \sigma_{n}^{i}\right)$. These show the expected behavior, namely a tendency to approach the expected boundary spin values at chain ends, e.g., $\left\langle\sigma_{N}^{y}\right\rangle \rightarrow$ $\sigma_{L}^{y}=-0.8$ etc. (data not shown).

The current dependence on $\Delta$ for different sizes $N \leqslant 10$ is shown in Fig. 1. First, we see the antisymmetry of $J_{z}(\Delta)$ with respect to $\Delta$ for odd $N$, in accordance with (10). Second, we note that the flux $J_{z}(0)=0$ for all $N>2$ in the freefermion case $\Delta=0$. In this respect, we remark that (i) the above property is not true for the other current components, and (ii) the vanishing of the $J_{z}$ current is very likely to be due to the absence of $\sigma_{z}$ gradient induced from boundaries (due to our choice of Lindblad operators) and to the lack of corresponding interaction terms in the Hamiltonian. Moreover, for the case of odd $N$, the vanishing of $J_{z}$ is rigorously proved, being a direct consequence of Eq. (10). We also note that the gradients of $\partial J_{z}(\Delta) / \partial \Delta$ at $\Delta=0$ seem to depend only on parity of $N$, apart from the nongeneric cases $N=2,3$, i.e., $\left(J_{z}\right)^{\prime}(0)=\alpha_{-}<0$ for even $N$ and $\left(J_{z}\right)^{\prime}(0)=\alpha_{+}>0$ for odd $N$, where $\alpha_{ \pm}$(at least numerically) is independent of $N$.

At large anisotropy values $\Delta \rightarrow \pm \infty$, the flux $J_{z}$ tends to zero. This can be demonstrated in the following way: For infinitely large $\Delta$, the system evolution is described essentially by Heisenberg equations of motion for an Ising Hamiltonian $H_{I}=\sum_{n} \sigma_{n}^{z} \sigma_{n+1}^{z}$, i.e., $\frac{\partial \rho}{\partial t}=-\frac{i}{\hbar}\left[H_{I}, \rho\right]$, the general timeindependent solution of which is given by $\rho_{I}=\sum \alpha_{k}|k\rangle\langle k|$, where $|k\rangle$ are eigenstates of the $H_{I}$, i.e., the set of all diagonal matrices in the representation where $\sigma^{z}$ is diagonal. The flux 


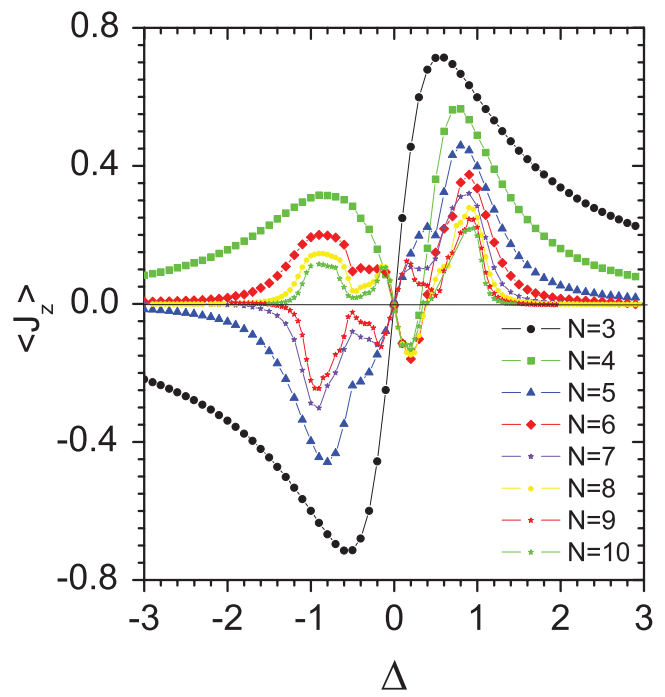

FIG. 1. (Color online) Stationary spin currents $J_{z}$ vs $\Delta$ for the $\mathcal{H}_{X X Z}$ chain of different lengths $N \leqslant 10$ with $\Gamma=4$. Symbols refer to numerical results obtained from direct integrations of the Lindblad master equation, while joining lines are drawn only as a guide for the eyes.

$J_{z}$ in this representation is a difference of the two off-diagonal elements of $\rho_{I}$ and is therefore trivially 0 . For large but finite $\Delta$, it is tempting to relate the exponentially small current to the phenomenon of "edge-locking" [19] observed in the quantum evolution of the $X X Z$ chain without Lindblad operators. In that case, there are blocks of spins pointing in the $z$ direction that are almost immobile in the vicinity of the boundary and hence suppress a magnetization current. Apparently, the coupling to an environment described by the Lindblad operators is not sufficient to significantly overcome edge-locking.

Another sign of an intrinsic interplay between the local action of Lindblad operators and bulk behavior governed by the Hamiltonian $H$ is seen if we look at the scaling of the current $J_{z}$ with system size $N$. In the region $|\Delta| \gtrsim 1$, the $J_{z}$ decreases as $J_{z} \sim \exp (-\gamma N)$ for fixed $\Delta$, while in the region $|\Delta| \lesssim 1$ the flux decreases algebraically as $\max J_{z} \sim 1 / N$,

$$
J_{z}(N) \sim\left\{\begin{array}{lll}
\exp (-\gamma N) & \text { for } & |\Delta|>1, \\
1 / N & \text { for } & |\Delta|<1 .
\end{array}\right.
$$

Notice that in this case we look at the absolute maxima of the current; see Fig. 2. Since in the infinite system $N=\infty$ the region $|\Delta|<1(|\Delta|>1)$ corresponds to the critical gapless phase with algebraically decaying correlations (gapped phase with exponentially decaying correlations), it is natural to suppose that the behavior of the current $J_{z}$ induced by the local pumping is influenced by the critical properties of the Hamiltonian. It is quite remarkable that one can see the signs of the bulk behavior already at small system sizes $N \leqslant 10$. With the help of the quasiclassical approximation presented in the next section, which turns out to work qualitatively well in the $|\Delta| \gtrsim 1$ region, we can arrive at much larger system sizes and confirm our conclusions. Note that transport properties of integrable systems are often anomalous, e.g., characterized by a ballistic spin and heat transport [20]. This corresponds to finite Drude weights, which sometimes can be computed analytically $[6,21]$. We remark that in our case
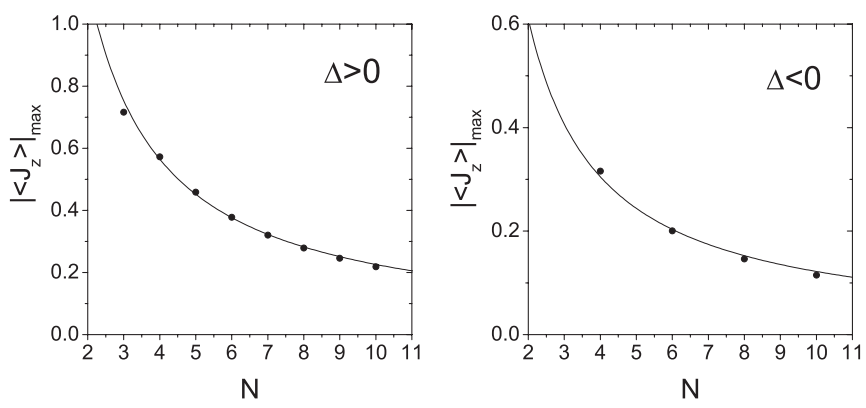

FIG. 2. Maxima of the $\left|\left\langle J_{z}\right\rangle\right|$ currents depicted in Fig. 1 vs $N$ for the range $\Delta>0$ (left panel) and $\Delta<0$ (right panel). Continuous curves show that the scaling follows a law of the type $a / N$ with $a=2.285$ for the left panel and $a=1.24$ for the right panel. Other parameters are fixed as in Fig. 1. The maxima of the current modulus for odd- $N$ values have not been displayed in the right panel because, due to the symmetry relations discussed in Sec. III, they are the same (and obviously follow the same scaling) as in the left panel.

the nonequilibrium steady states are characterized by at most diffusive transport [see Eq. (11)], in contrast to what was recently reported for the $X X Z$ Heisenberg model with a different realization of boundary reservoirs (two Lindblad operators at each system end, not optimized for maximal transport) [22]. In particular, from the data depicted in Fig. 1 it follows that the scaling at the isotropic point $\Delta=1$ follows the same scaling in (11) for the critical (gapless) region (with a prefactor $a \approx 2$ ), in contrast with the scaling $1 / \sqrt{N}$ obtained in Ref. [22] (this being another example of the influence of boundary reservoirs on bulk properties).

Another remarkable observation can be made if we look at the signs of the flux $J_{z}$ in the $\Delta<0$ region. This sign respects the parity of $N: \operatorname{sgn}\left(J_{z}(N)\right)=(-1)^{N}$. An explanation of this phenomenon is related to the alternating structure of the stationary profiles $\left\langle\sigma_{n}^{y}\right\rangle,\left\langle\sigma_{n}^{z}\right\rangle$, as illustrated in Fig. 3. It is worth pointing out that a perturbation theory in the small parameter $\varepsilon=\Delta^{-1} \ll 1$ around the point $\Delta=-\infty$, which could in principle explain the phenomenon of flux alternation, is difficult to build for the following reason: The scaling $J_{z}(N) \sim \exp (-\gamma N)$ for fixed $\Delta$ implies a scaling $\left|J_{z}(\Delta)\right| \sim 1 /|\Delta|^{N-\gamma}=\varepsilon^{N-\gamma}$ for fixed $N$. From the numerical data, we estimate $\left|J_{z}(\Delta)\right| \approx C /|\Delta|^{N-3 / 2}=C \varepsilon^{N-3 / 2}$. So the first nonvanishing order of perturbation theory (at least for the quantity $J_{z}$ in which we are interested) is $\varepsilon^{N-3 / 2}$, where the $N$ is the system size. On the other hand, in the opposite limit $\Delta \rightarrow-0$, the analytical treatment might be possible, because at $\Delta=0$ the Hamiltonian $H$ becomes a free-fermion Hamiltonian. Several analytic results are available for the Lindblad master equation at the free-fermion point [14,23-26]; however, these results are not applicable to our choice of the Lindblad operators (7).

We have studied the dependence of the flux $J_{z}$ on various parameters, including the coupling strength $\Gamma$. At large coupling strength, one expects an effective freezing of the hoppings of the boundary spins due to the quantum Zeno effect. However, unlike in the setting [3], where a quantum Zeno effect suppresses the magnetization current, for our choice of the Lindblad operators the current suppression is not observed; see Fig. 4. The reason is that through large couplings we "freeze" 

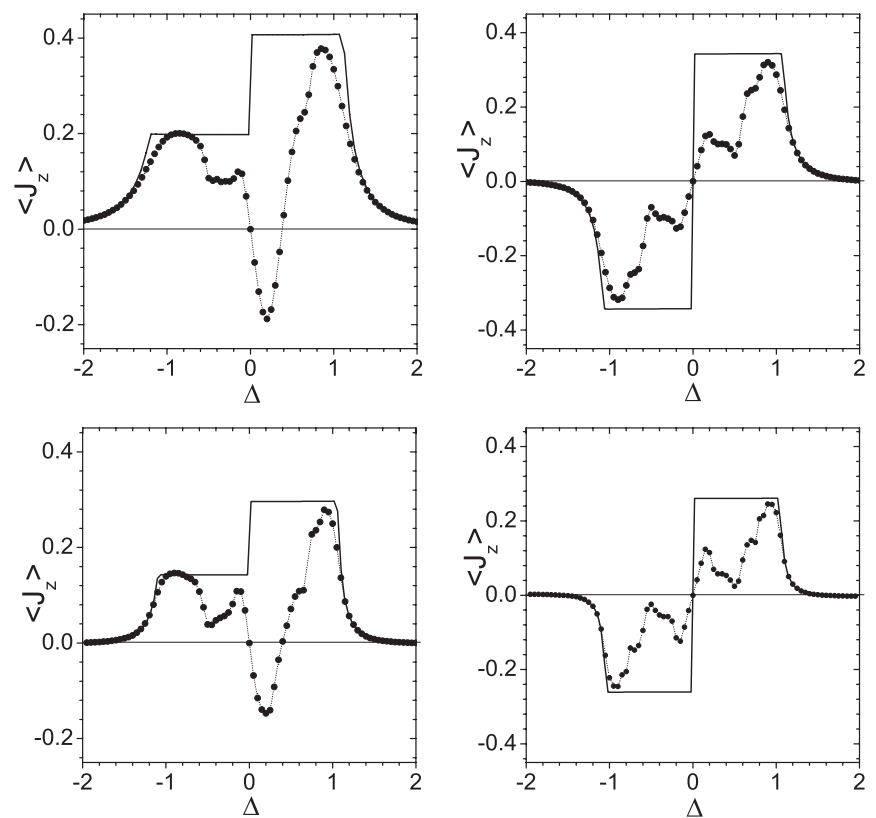

FIG. 3. Comparison between quantum (dots joined by dotted lines) and semiclassical (continuous lines) mean currents $\left\langle J_{z}\right\rangle$ vs $\Delta$ for $\Gamma=4$ and $N=6,7$ (top left and right panels, respectively) and $N=8,9$ (corresponding bottom left and right panels).

the boundary $\sigma^{y}$ and $\sigma^{x}$ spin components, while hoppings in the $\sigma^{z}$ component, contributing to the current $J_{z}$, may still occur.

\section{MEAN-FIELD APPROACH}

Studies of the quantum system dynamics via solving the full system (1) or by an appropriate Monte Carlo scheme are inevitably restricted to small system sizes $N$ due to exponentially growing complexity. It is therefore desirable to complement them with other approaches that might help to study a given specific feature of the quantum system and advance our understanding. It turns out that the simple mean-field approach described below captures the quantities we are interested in (one-point correlation functions and flux $J_{z}$ ) with very good precision for large values of $\Delta$ and correctly reproduces the alternation of the sign of the current with $N$. Also, in spite of the very qualitative behavior found inside the $\Delta<1$ region, the main $J_{z}$ peaks in this region are predicted reasonably well (see Fig. 3 and the discussion below).

Since the complexity in the mean-field approach grows algebraically with the size (rather than exponentially), we can easily handle much larger system sizes than in the quantum case. Neglecting correlations, i.e., setting $\left\langle\sigma_{n}^{x} \sigma_{n+1}^{z}\right\rangle=$ $\left\langle\sigma_{n}^{x}\right\rangle\left\langle\sigma_{n+1}^{z}\right\rangle$, we write down the set of equations of motion for spin operators $\left\langle\sigma_{n}^{x}\right\rangle,\left\langle\sigma_{n}^{y}\right\rangle,\left\langle\sigma_{n}^{z}\right\rangle$ :

$$
\begin{aligned}
& \frac{d}{d t} \sigma_{n}^{y}=\Delta \sigma_{n-1}^{z} \sigma_{n}^{x}-\sigma_{n-1}^{x} \sigma_{n}^{z}+(n-1 \rightarrow n+1), \\
& \frac{d}{d t} \sigma_{n}^{z}=-\sigma_{n-1}^{y} \sigma_{n}^{x}+\sigma_{n-1}^{x} \sigma_{n}^{y}+(n-1 \rightarrow n+1), \\
& \frac{d}{d t} \sigma_{n}^{x}=\sigma_{n-1}^{y} \sigma_{n}^{z}-\Delta \sigma_{n-1}^{z} \sigma_{n}^{y}+(n-1 \rightarrow n+1)
\end{aligned}
$$

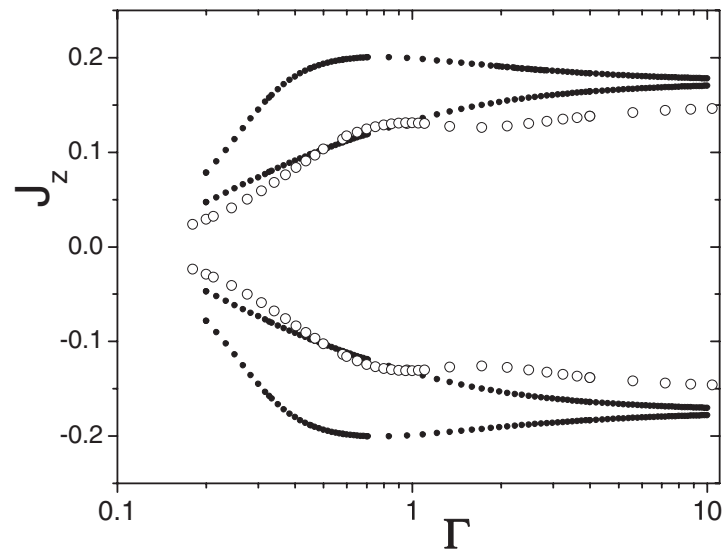

FIG. 4. Mean-field (black dotted curves) and quantum (open circles) numerical solutions vs the coupling parameter $\Gamma$ for a chain of $N=5$ sites and for two opposite values of $\Delta: \Delta=1.5\left(J_{z}>0\right.$ curves) and $\Delta=-1.5$ ( $J_{z}<0$ curves $)$.

in the bulk $n=2,3, \ldots, N-1$, and at the boundaries $(n=1)$,

$$
\begin{aligned}
& \frac{d}{d t} \sigma_{n}^{y}=\Delta \sigma_{n+1}^{z} \sigma_{n}^{x}-\sigma_{n+1}^{x} \sigma_{n}^{z}-\Gamma_{y}\left(\sigma_{n}^{y}-\sigma_{L}^{y}\right), \\
& \frac{d}{d t} \sigma_{n}^{z}=-\sigma_{n+1}^{y} \sigma_{n}^{x}+\sigma_{n+1}^{x} \sigma_{n}^{y}-\Gamma_{z}\left(\sigma_{n}^{z}-\sigma_{L}^{z}\right), \\
& \frac{d}{d t} \sigma_{n}^{x}=\sigma_{n+1}^{y} \sigma_{n}^{z}-\Delta \sigma_{n+1}^{z} \sigma_{n}^{y}-\Gamma_{x}\left(\sigma_{n}^{x}-\sigma_{L}^{x}\right),
\end{aligned}
$$

where $\Gamma_{i}$ and $\sigma_{L}^{i}$ are given by the expressions (5). Analogously, we write down the mean-field equations for the right boundary $n=N$. Solving them numerically for the stationary state, we find qualitative agreement with the quantum problem at least in part of the parameter range (see Fig. 3).

Note that for $|\Delta|>1$, we found also a qualitative agreement for the flux $J_{z}$, which becomes more accurate as $N$ or $\Delta$ grows. To explain this agreement, we note that the current $J_{z}$ can be written as a sum of the disconnected and the connected part,

$$
J_{n, z}=\left\langle\sigma_{n}^{x}\right\rangle\left\langle\sigma_{n+1}^{z}\right\rangle-\left\langle\sigma_{n}^{y}\right\rangle\left\langle\sigma_{n+1}^{x}\right\rangle+\left\langle\sigma_{n}^{x} \sigma_{n+1}^{z}\right\rangle_{c}-\left\langle\sigma_{n}^{y} \sigma_{n+1}^{x}\right\rangle_{c},
$$

the contribution of the latter being neglected in the mean-field approach. In the full quantum system, one observes a drastic decrease of the "weight" of the connected part outside the critical region $|\Delta|<1$. Thus, outside the critical region, the current $J_{z}(\Delta)$ is determined essentially by one-point correlations, thus validating the mean-field assumption.

The alternation of the sign of the flux $J_{z}(N)$ with $N$ within the mean-field description is a consequence of the oscillatory behavior of the one-point functions $\left\langle\sigma_{n}^{z}\right\rangle$ and $\left\langle\sigma_{n}^{z}\right\rangle$; see Fig. 5. We expect, however, this oscillatory behavior to govern the flux alternation also in the quantum case for $\Delta \ll-1$, where the flux $J_{z}$ is determined, essentially, by one-point correlations (data not shown).

Quite remarkably, in the mean-field description we find two different stationary solutions $\rho$ for a given value of $\Delta$ and in the whole parameter range (see the black dotted curves in Fig. 4), which are characterized by different fluxes $J_{z}$ and differ by the sign of the average $z$ magnetization $M^{z}=\sum_{n}\left\langle\sigma_{n}^{z}\right\rangle$. For positive $\Delta$, these solutions can be associated with the 

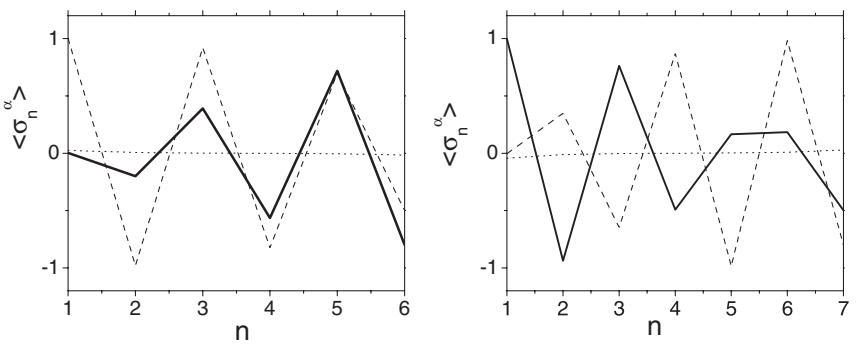

FIG. 5. Magnetization profiles $\left\langle\sigma_{n}^{x}\right\rangle,\left\langle\sigma_{n}^{y}\right\rangle,\left\langle\sigma_{n}^{z}\right\rangle$ shown with broken, solid, and dotted lines, respectively. The mean-field flux between sites 1 and 2 is given by $J_{1}^{z}=\sigma_{1}^{y} \sigma_{2}^{x}-\sigma_{1}^{x} \sigma_{2}^{y} \approx-\sigma_{2}^{y}$, because $\sigma_{1}^{x} \approx \sigma_{L}^{x}=1, \sigma_{1}^{y} \approx \sigma_{L}^{y}=0$. Note that $\sigma_{n}^{y}$ is alternating its sign with $n$ and tends to fixed (independent of $N$ ) boundary values $\sigma_{L}^{y}$ and $\sigma_{R}^{y}$. Therefore, an increase of the system size $N \rightarrow N+1$ changes the sign of $\sigma_{2}^{y}$, which entails the change of the sign of the flux $J_{z}$; compare the left panel $(N=6)$ and the right panel $(N=7)$. The above arguments are valid also for the quantum (computed from the Lindblad equation) case for $\Delta<-1$, where the flux $J_{z}$ is determined essentially by one-point correlation functions.

first $\lambda_{1} \approx N / 2$ and the second $\lambda_{2}=\lambda_{1} / 2$ harmonics in the respective $\left\langle\sigma_{n}^{y}\right\rangle,\left\langle\sigma_{n}^{x}\right\rangle$ density profiles (see Fig. 6). We remark that the mean-field solutions with larger absolute $J_{z}$ are more difficult to obtain from random initial conditions due to smaller attraction basins. Also, the specular symmetry of the meanfield curves around the $J_{z}=0$ axis clearly indicates that the odd- $N$ symmetry of the quantum problem extends to the meanfield model as well.

It is also worth noting that the (apparently single) quantum solution for a fixed value of $\Delta$ in Fig. 4 (open circles) closely follows and partially overlaps the mean-field solution with smaller flux $J_{z}$, while the (possibly) missing second quantum solution is the one corresponding to the classical curve with larger flux and a smaller basin of attraction (this last fact could explain the failure of all our attempts to find this solution). However, the possibility of the existence of multiple stationary solutions in the quantum case cannot be excluded, and more investigations are required to clarify the issue. In this respect, we remark that, apart for the very special case of Lindblad operators taken as eigenoperators of the Hamiltonian $H$ (this being not our case), there is no general proof, to the best of our knowledge, of the uniqueness of the solution of the Lindblad master equation (in the mentioned special case, the evolution equations for the populations of the energetic levels become the classical master equation with a stochastic matrix, and the uniqueness of the solution follows from the Perron-Frobenius theorem). Considering the very good agreement observed between mean-field and quantum results in the $|\Delta|>1$ region, the possibility that multiple stationary solutions could exist also in the quantum case should not be overlooked.

\section{CONCLUSION}

In summary, we have studied the magnetization current induced in the one-dimensional quantum $X X Z$ spin chain by magnetic boundary fields and showed that this current can be controlled and maximized by appropriate boundary magnetic
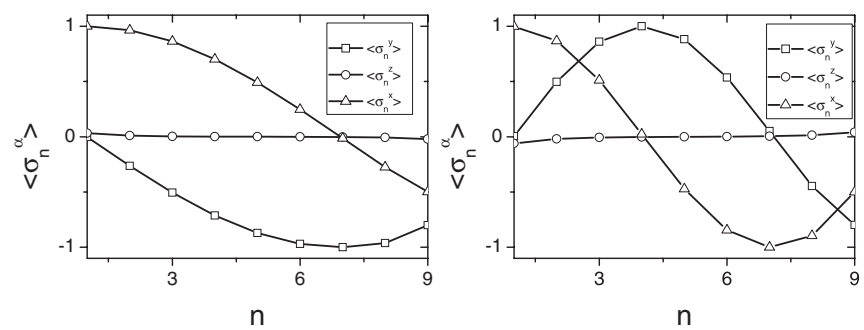

FIG. 6. The left panel shows magnetization profiles of $\left\langle\sigma_{n}^{\alpha}\right\rangle$ for two stationary mean-field solutions. Parameters: $N=9, \Delta=0.52$. The solution shown in the right panel corresponds to negative $J_{z}$ and is "quantistically unstable." The quantum stable solution (left panel) carries positive flux $J_{z}$. The profile corresponds to positive $J_{z}$ and $N=9$.

fields. The dependence of the current on the anisotropy parameter $\Delta$ and on the boundary field strengths has been characterized analytically and numerically both by means of system symmetries and by the numerical evaluation of the stationary density matrix in the framework of the Lindblad master equation. We have shown that for odd system sizes $N$, the existence of additional symmetries in the system causes the current to become an odd function of $\Delta$. For arbitrary sites and for $\Delta$ negative, the current was also shown to alternate its sign with the system size, $\operatorname{sgn}\left(J_{z}(N)\right)=(-1)^{N}$. The scaling properties of the current with the system size have been characterized both in the critical and in the gapped regions.

We found that in the critical region, $-1<\Delta<1$, the current scales as $J_{z}(N) \sim 1 / N$ while in the gapped region it scales as $J_{z}(N) \sim \exp (-\alpha N)$. The behavior of the magnetization conductivity in the critical region shows a nonmonotonic behavior with the existence of relative maxima at intermediate values and with the current always vanishing for $\Delta=0$. This behavior is notably different from the free quantum evolution of an $X X Z$ chain without boundary fields but with a step initial state with opposite magnetizations $[27,28]$. In that case, a nonequilibrium state with a current of order 1 is attained even when $\Delta=0$. A simple mean-field approach neglecting nearest-neighbor correlations predicts qualitatively well the value of $J_{z}(N)$ for the gapped region and the value of the absolute maximum in the critical region.

The existence of two different stationary solutions for the mean field $\rho$ in the whole parameter range has been demonstrated, and the possibility that multiple stationary solutions could exist also in the quantum case has been suggested. This last possibility represents a very interesting problem that deserves further investigations.

\section{ACKNOWLEDGMENTS}

V.P. acknowledges the University of Salerno for a research grant to support this work. M.S. acknowledges support from the Ministero dell' Istruzione, dell' Università e della Ricerca (MIUR) through a Programma di Ricerca Scientifica di Rilevante Interesse Nazionale (PRIN) initiative. 
[1] H.-P. Breuer and F. Petruccione, The Theory of Open Quantum Systems (Oxford University Press, Oxford, 2002).

[2] M. B. Plenio and P. L. Knight, Rev. Mod. Phys. 70, 101 (1998).

[3] G. Benenti, G. Casati, T. Prosen, and D. Rossini, Europhys. Lett. 85, 37001 (2009).

[4] F. Heidrich-Meisner, A. Honecker, and W. Brenig, Eur. Phys. J. Special Topics 151, 135 (2007), and references therein.

[5] X. Zotos, J. Phys. Soc. Jpn. Suppl. 74, 173 (2005), and references therein.

[6] B. S. Shastry and B. Sutherland, Phys. Rev. Lett. 65, 243 (1990).

[7] X. Zotos, Phys. Rev. Lett. 82, 1764 (1999); J. Benz, T. Fukui, A. Klümper, and C. Scheeren, J. Phys. Soc. Jpn. Suppl. 74, 181 (2005).

[8] T. Antal, Z. Rácz, and L. Sasvári, Phys. Rev. Lett. 78, 167 (1997).

[9] T. Antal, Z. Rácz, A. Rákos, and G. M. Schütz, Phys. Rev. E 57, 5184 (1998).

[10] X. Zotos and P. Prelovsek, Phys. Rev. B 53, 983 (1996).

[11] B. N. Narozhny, A. J. Millis, and N. Andrei, Phys. Rev. B 58 R2921 (1998).

[12] P. Prelovšek, S. El Shawish, X. Zotos, and M. Long, Phys. Rev. B 70, 205129 (2004).

[13] J. V. Alvarez and C. Gros, Phys. Rev. Lett. 88, 077203 (2002).

[14] T. Prosen, New. J. Phys. 10, 043026 (2008).
[15] V. Popkov and M. Salerno, Phys. Rev. A 71, 012301 (2005); V. Popkov, M. Salerno, and G. Schütz, ibid. 72, 032327 (2005).

[16] V. Popkov and M. Salerno, Europhys. Lett. 84, 30007 (2008); M. Salerno and V. Popkov, Phys. Rev. E 82, 011142 (2010); Acta Appl. Matem. 115, 75 (2011).

[17] P. Krammer, H. Kampermann, D. Bruss, R. A. Bertlmann, L. C. Kwek, and C. Macchiavello, Phys. Rev. Lett. 103, 100502 (2009).

[18] M. Cramer, M. B. Plenio, and H. Wunderlich, Phys. Rev. Lett. 106, 020401 (2011).

[19] M. Haque, Phys. Rev. A 82, 012108 (2010).

[20] X. Zotos, F. Naef, and P. Prelovsek, Phys Rev. B 55, 11029 (1997).

[21] A. Klümper and K. Sakai, J. Phys. A 35, 2173 (2002).

[22] M. Žnidarič, Phys. Rev. Lett. 106, 220601 (2011).

[23] M. Žnidarič, J. Stat. Mech. (2010) L05002.

[24] M. Žnidarič, J. Phys. A 43, 415004 (2010).

[25] D. Karevski and T. Platini, Phys. Rev. Lett. 102, 207207 (2009).

[26] T. Prosen and M. Žnidarič, Phys. Rev. Lett. 105, 060603 (2010).

[27] T. Antal, Z. Rácz, A. Rákos, and G. M. Schütz, Phys. Rev. E 59, 4912 (1999).

[28] D. Gobert, C. Kollath, U. Schollwöck, and G. M. Schütz, Phys. Rev. E 71, 036102 (2005). 